\title{
Desafios da acreditação em um hospital da rede Fhemig
}

\section{Diego Resende Martins.}

Graduado em Fisioterapia pela Pontifícia Universidade Católica de Minas Gerais. Graduando em Gestão de Serviços de Saúde pela Universidade Federal de Minas Gerais.

Email:drmmartins@yahoo.com.br

\section{Emiza Macedo Rates}

Graduada em Enfermagem pela Universidade Federal de Alagoas. Gerente Administrativa do Hospital Infantil João Paulo II, Fundação Hospitalar de Minas Gerais - FHEMIG.

Email: emiza.rates@gmail.com

\section{Kênia Lara Silva}

Doutora e mestre em Enfermagem pela Universidade Federal de Minas Gerais. Professora adjunta da Escola de Enfermagem da Universidade Federal de Minas Gerais.

Email: kenialara17@yahoo.com.br

\section{Caroline Gonçalves Ribeiro de Almeida}

Graduada em Terapia Ocupacional pela Universidade Presidente Antônio Carlos. Graduada em

Enfermagem pela Universidade Salgado de Oliveira.

Email: caroline_gra@yahoo.com.br

\section{Thereza Raquel Ribeiro Senra}

Graduada em Enfermagem pelo Centro Universitário Metodista Izabela Hendrix. Consultora autônoma em aleitamento materno.

Email: therezasenra@yahoo.com.br

\section{Rafael Luiz Pinho Teixeira Mourão}

Graduado em Enfermagem pela Universidade Salgado de Oliveira.

Email: rafaelmourao@ig.com.br

RESUMO: Trata-se de um relato de experiência sobre a implantação da gestão de qualidade em instituições de saúde. O objetivo é apresentar as potencialidades e dificuldades no processo de implantação e gestão do Sistema de Gestão da Qualidade de um hospital da Rede FHEMIG. Os resultados indicam que, entre as potencialidades para a implantação do Sistema de qualidade, estão o envolvimento da gerência administrativa e a democratização das relações hierárquicas permitindo a participação das diferentes gerências no processo. Citam-se também o apoio do nível central da FHEMIG e a consolidação do Escritório da Qualidade. Em relação aos desafios, podem ser apontados a dificuldade dos trabalhadores para incorporar o uso das ferramentas em seu cotidiano de trabalho e os processos específicos inerentes a uma instituição pública como, por exemplo, o excesso de burocracia para se promoverem mudanças estruturais. Conclui-se que a definição politico-institucional assumida pela FHEMIG foi imprescindível para a melhoria da qualidade no Hospital e tem permitido a reorganização dos processos de trabalho.

PALAVRAS-CHAVE: Gestão da Qualidade; Acreditação/ instituições de saúde;

\section{Challenges in accreditation of a Fhemig hospital}

ABSTRACT: It is an experience report on the implementation of quality management in health institutions. The goal is to present the potential and difficulties in the implementation and management of the Quality Management System in a FHEMING networking hospital. The results indicate that among the potential to implement the quality system is the involvement of administrative management and democratization of the hierarchical relationships in empowering different managers in the process. It also cites the support of the central FHEMIG and consolidation of the Office of Quality. Regarding the challenges, can be pointed out the difficulty of workers incorporated the use of tools in their daily work and the specific processes involved in a public institution such as red tape for structural changes. It is concluded that the definition assumed by the political-institutional FHEMIG was essential for improving quality in the hospital and has allowed the reorganization of work processes.

KEYWORDS: Quality Management, Accreditation / health institutions; 


\section{INTRODUÇÃO}

A gestão de qualidade em instituições de saúde tem se tornado imprescindível para a sustentabilidade das mesmas. A temática está presente no campo privado de forma mais intensa, determinada pela competição entre as empresas. Contudo, as instituições públicas também estão investindo em melhoria de qualidade, em resposta a movimentos de racionalização de custos e, ao mesmo tempo, de ampliação da noção de segurança e de direitos dos usuários. (FERREIRA, 1999; CORNETA; FELICE, 1994)

O termo qualidade, na área da administração, é definido como estratégia de gestão em que se procura otimizar a produção e reduzir os custos financeiros, humanos, etc (HOUAISS, 2001, p. 2345). Originalmente o conceito foi desenvolvido para aplicação na indústria americana, na década de 1950, com seu apogeu na indústria automobilística japonesa na década de 1960 (D'INNACENZO et al., 2006).

$\mathrm{Na}$ área da saúde, os primeiros relatos de estratégias para melhorar o resultado dos serviços datam da década de 1920, com o Colégio Americano de Cirurgiões - CAC, no Hospital Geral de Massachusetts vinculado à Universidade de Harvard, onde foi estabelecido o Programa de Padronização Hospitalar PPH. Embora naquele momento ainda não estivesse estabelecido como Programa de Qualidade, a análise do conteúdo do PPH revela sua direcionalidade para as condições necessárias para os procedimentos médicos com corpo funcional habilitado. (FELDMAN et al., 2005; NOVAES, 2007)

O advento da Segunda Guerra Mundial foi decisivo para o movimento da qualidade nos Estados Unidos e no Canadá. A amplitude que o Manual da PPH tinha adquirido tornou-se obsoleta em face da elevação dos custos, da sofisticação crescente da assistência médica, do aumento do número de instituições a serem padronizadas e da complexidade e da grande procura de especialidades não cirúrgicas. A partir daí o CAC começou a desenvolver parcerias com outras associações médicas americanas e canadenses para apoio e participação com relação à melhoria e à promoção da acreditação voluntária. Em 1951, cria-se, então, a Comissão Conjunta de Acreditação de Hospitais e, em 1952, estabelecem oficialmente a Joint Comission on Acreditation of Hospitals - JCAH que, em 1987, passa a ser denominada de Joint Comission on Acreditation of Healthcare Organizations - JCAHO. (FELDMAN et al., 2005)

No Brasil, as experiências com Programas de Qualidade são relativamente recentes e são desenvolvidas especialmente em instituições hospitalares privadas. Instituições como o Instituto do Coração da Faculdade de Medicina da USP, na década de 1970, o Hospital Israelita Albert Einstein em São Paulo, na década de 80, Santa Casa de Misericórdia em Porto Alegre, na década de 1980 e a Unidade de Cardiologia do Hospital da Universidade Federal de São Paulo - UNIFESP, na década de 1990 adotaram modelos de gestão baseados na qualidade o que proporcionou ganho com relação aos recursos humanos, garantindo a satisfação do cliente interno em seu ambiente de trabalho, a satisfação do paciente com o atendimento a suas necessidades, bem como modificações nos indicadores de estatística hospitalar (BALSANELLI; JERICÓ, 2005).

No processo de avaliação e certificação de qualidade nas instituições, construiram-se padrões de acreditação. Nesse sentido, a Acreditação é o procedimento de avaliação dos recursos institucionais, voluntário, periódico, reservado e sigiloso, que tende a garantir a qualidade da assistência por meio de padrões previamente aceitos. (FELDMAN et al., 2005). Segunda as autoras, os padrões de acreditação podem variar de mínimos (definindo o piso ou base) até aqueles mais elaborados e exigentes, que definem diferentes níveis de satisfação e qualificação.

O processo de Acreditação, no Brasil, começa a tornar-se possível no início da década de1990, com a publicação da Acreditação de Hospitais para a América e Caribe. Em 1990, foi firmado um convênio entre a Organização Pan-americana de Saúde - OPAS e a Federação Latino-americana de Hospitais para a produção de um Manual de "Padrões de Acreditação para a América Latina", estruturado em padrões e níveis de complexidade, sendo que a avaliação final é determinada pelo nível mínimo atingido. Em 1992, foi realizado, em Brasília, o I Seminário Nacional de Acreditação, com a participação de representantes de diversas entidades nacionais da área da saúde, sendo apresentado o Manual de Acreditação (NOVAES; PAGANINI, 1994).

Em 1998, foi elaborada uma nova versão do Manual de Acreditação e discutidos as normas técnicas que regulamentam o papel do órgão acreditador, a relação entre a instituição acreditadora e o Ministério da Saúde, o código de ética e o perfil do avaliador. Em agosto de 1999, a Organização Nacional de Acreditação - ONA - passou a ser responsável pelo estabelecimento de padrões e monitoramento do processo de acreditação realizado pelas instituições acreditadoras. Assim, inicia-se oficialmente, no país, o processo de Acreditação em 2000 (BRASIL, 2004). 
A Acreditação Hospitalar configura-se como uma metodologia desenvolvida para apreciar a qualidade da assistência oferecida em todos os serviços de um hospital. Tem como base a avaliação dos padrões de referência desejáveis e indicadores mínimos de qualidade previamente divulgados em um Manual construído por peritos da área. A solicitação da Acreditação pela instituição é um ato voluntário, periódico, espontâneo, reservado e sigiloso em que se pretende obter a condição de acreditada de acordo com padrões previamente aceitos. É escolhida a instituição acreditadora que desenvolverá o processo de certificação (BRASIL, 2004).

Ao contrário dos processos tradicionais de avaliação, a acreditação possui uma abordagem educativa, baseada na reflexão da prática profissional, que leva à elaboração de padrões de excelência de desempenho. Por ser um processo, sobretudo reflexivo, sempre revela novas formas de visualizar e agir sobre os problemas da instituição (ROONEY; OSTENBER, 1999).

Ao aderir ao processo de acreditação, a organização prestadora de serviços de saúde firma sua responsabilidade e seu comprometimento com a segurança, com a ética profissional, com a eficiência dos procedimentos que realiza e com a garantia da qualidade do atendimento à população (ONA, 2000).

O movimento da acreditação tem impulsionado um grande número de instituições a se submeterem ao processo. Em 2012, segundo dados da Organização Nacional de Acreditação demonstrados na Figura 1 , há, no Brasil, cerca de 292 organizações de saúde com certificação de qualidade que recebem o título de Acreditadas, Acreditadas Plenas ou Acreditadas com Excelência conforme o cumprimento dos padrões de qualidade do Sistema Brasileiro de Acreditação (SBA).

Figura 1: Número de organizações acreditadas no Brasil e em Minas Gerais.

\begin{tabular}{|l|c|c|}
\hline Nível & $\begin{array}{c}\text { Número de } \\
\text { organizações no } \\
\text { Brasil }\end{array}$ & $\begin{array}{c}\text { Número de } \\
\text { organizações em } \\
\text { Minas Gerais }\end{array}$ \\
\hline Acreditado & 89 & 4 \\
\hline Acreditado Pleno & 93 & 25 \\
\hline Acreditado com excelência & 110 & 26 \\
\hline TOTAL & 292 & 55 \\
\hline
\end{tabular}

Fonte: Organização Nacional de Acreditação, janeiro de 2012.

Em Minas Gerais, os dados da Organização Nacional de Acreditação, em janeiro de 2012, revelam a existência de 55 organizações acreditadas em um universo de 712 organizações de saúde. Isso revela que ainda há a necessidade de se investir cada vez mais em gestão da qualidade visando a melhoria contínua da prestação dos serviços aos usuários/clientes e das condições de trabalho dos colaboradores (ONA, 2012).

Chama a atenção o fato de que a maior parte das instituições é de natureza privada. Contudo, há iniciativas de investimento na acreditação em instituições públicas, como o Hospital Regional Antônio Dias, de Patos de Minas, o primeiro da Rede FHEMIG a ser acreditado. (ONA, 2012).

A obtenção da qualidade é um processo complexo. Segundo Ferreira (1999), para o setor público, esse processo exige maior esforço de mudança uma vez que, tradicionalmente, esse setor esteve caracterizado por modelos de gestão burocráticos e tayloristas. As mudanças, nesse sentido, vão além de oferecer um serviço público de qualidade, mas também envolvem diminuição de gastos do orçamento, descentralização, inovações nos processos seletivos .

Para isso, o setor público precisa ter uma estrutura mais flexível e adaptável ao contexto atual, capaz de atender as necessidades e as expectativas de seus clientes-cidadãos. O que se espera de uma organização pública e de seus funcionários não é um serviço provido de documentos para solicitação e atendimento de uma necessidade, mas sim uma organização de funcionários capazes de resolver os problemas do cidadão, colocando-o em primeiro plano, tratando-o com seriedade e respeito acima de tudo. Caso contrário, o setor público dificilmente irá projetar uma imagem coerente com essa nova sociedade. (LAS CASAS, 1999)Há diferenças entre o setor público e o privado que devem ser levadas em consideração quando se fala de qualidade: para Las Casas (1999), enquanto o setor privado está baseado no interesse, na capacidade de pagar pelo serviço prestado, o setor público está alicerçado no dever, pois precisa servir a todos igualmente (LAS CASAS,1999). Ferreira (1999) aponta também que a motivação dos dirigentes, 
caracterizada pela competitividade nas empresas privadas e pela reeleição/ permanência no cargo no setor público, também é um fator a ser considerado. Ainda, devemos atentar para os modelos decisórios que tendem à centralização nas instituições públicas.

Com isso, ao pensar em qualidade no serviço público, parecem surgir desafios. Assim, o objetivo deste trabalho é analisar os desafios no processo de implementação do Sistema de Gestão da Qualidade em uma instituição pública. Tomou-se como cenário, para o relato da experiência, um hospital da Rede Fundação Hospital do Estado de Minas Gerais - FHEMIG.

\section{A FHEMIG E O MOVIMENTO DA QUALIDADE}

A Fundação Hospitalar do Estado de Minas Gerais foi criada em 1977, está vinculada à Secretaria de Estado de Saúde de Minas Gerais (SES) e é prestadora de serviços de complexidade secundária e terciária, exclusivamente, para o Sistema Único de Saúde (SUS). ${ }^{1}$

A Rede FHEMIG é uma das maiores gestoras de hospitais públicos do país e tem atuação em seis complexos assistenciais: urgência e emergência, especialidades, saúde mental, hospitais gerais, MG Transplantes, reabilitação e cuidado ao idoso. São 20 unidades assistenciais, sendo sete localizadas no interior do Estado de Minas Gerais e 13 na Região Metropolitana de Belo Horizonte, atuando na atenção ambulatorial e hospitalar nas áreas de urgência e emergência, saúde mental, reabilitação e cuidado ao idoso, oncologia, clínica médica, gineco-obstetrícia e neonatologia, infectologia, dermatologia e transplantes. Para isso, a Rede FHEMIG conta com 2.060 leitos operacionais, 221 leitos de UTI e 895 leitos asilares. Realiza, em média, 2.200 cirurgias (bloco cirúrgico) mensais, 869 cirurgias no bloco obstétrico, em média, por mês. Além disso, apresenta uma média de 5.500 internações/mês, 50.360 consultas médicas de urgência e 24.600 consultas especializadas programadas mensais. Os exames complementares correspondem a um montante de 323.300, número correspondente ao ano de 2009. ${ }^{2}$

A Fundação se inseriu em importantes processos históricos, como o marco zero da reforma psiquiátrica; a mudança de sanatórios, de ex-colônias para casas de saúde, voltadas para a reabilitação e cuidado ao idoso; a reintegração social de pacientes que antes eram isolados, como nos tratamentos da tuberculose e da hanseníase, entre outros.

Promoveu, ainda, inovações tecnológicas e incorporou novas técnicas assistenciais e equipamentos a suas unidades. Tornou-se referência e excelência nacionais em vários de seus serviços prestados à população.

A FHEMIG, em consonância com as diretrizes e políticas governamentais do Estado, e buscando cada vez mais contribuir para o desenvolvimento e o fortalecimento do Sistema Único de Saúde e para a inovação e incorporação tecnológica, tem adotado, em seu processo administrativo, técnicas e ferramentas modernas de gestão, incluindo a formulação de um planejamento estratégico para toda a Rede FHEMIG. Um Planejamento Estratégico, de maneira geral, busca dar o direcionamento às organizações face a seus diversos ambientes para que superem desafios, sobrevivam e antecipem-se às descontinuidades a que estão sujeitas. O Planejamento Estratégico de uma instituição pública ocorre de maneira semelhante, porém com suas especificidades. .Busca direcionar-se alinhada às políticas públicas a que se sujeita, sejam elas federais, estaduais e municipais e, ainda, àquilo que é a sua razão de existir: o seu negócio ou core business. Nesse sentido, o que norteia o Planejamento Estratégico de uma instituição como a FHEMIG são as Diretrizes do Governo do Estado de Minas Gerais, no caso o PMDI (Plano Mineiro de Desenvolvimento Integrado 2003; 2007) e as Políticas do SUS (PLANEJAMENTO ESTRATÉGICO FHEMIG, 2008).

O Planejamento Estratégico da FHEMIG está voltado, principalmente, para a melhoria da qualidade assistencial e do ambiente de trabalho de seus servidores e colaboradores. Todos os seus 25 objetivos norteadores nos mais diversos graus de amplitude (Pessoas, Processos, Comunidade e Minas Gerais), com exceção do objetivo referente à promoção de remuneração competitiva, estão alinhados com os níveis necessários para certificação da ONA em níveis 1, 2 e 3.

Assim, o Planejamento Estratégico reflete a preocupação de seus gestores em ofertar, aos usuários da Rede FHEMIG, serviços de alta qualidade e que sejam referência em suas mais diversas especialidades.

\footnotetext{
1 Disponível em: www.fhemig.mg.gov.br

2 Disponível em: www.fhemig.mg.gov.br
} 
No âmbito da FHEMIG, o processo de Acreditação se soma a outros em curso, como a implantação de Protocolos Clínicos, do Sistema de Gerenciamento de Custos, da Gestão do Conhecimento, da Gestão Estratégica, da Contratualização, da Gestão Ambiental, para dar sustentação ao Mapa Estratégico institucional. Nesse Mapa, estão estabelecidos os objetivos que permitem à Fundação enfrentar os desafios apontados na gestão hospitalar pública e perseguir a sua visão de futuro - "constituir-se em modelo de excelência no país para a promoção da assistência e qualificação hospitalar" (CARTILHA ACREDITAÇÃO FHEMIG, 2010).

\section{O SISTEMA DE GESTÃO DA QUALIDADE NO HOSPITAL- CENÁRIO}

O Sistema de Gestão da Qualidade do Hospital- Cenário baseia-se na padronização dos documentos de interesse nas áreas administrativa e assistencial, de modo a facilitar o desenvolvimento e o aperfeiçoamento dos processos, minimizando a utilização de recursos materiais, melhoria das condições de trabalho aos profissionais e maximizando os resultados.

Uma rede interna foi criada para que todos os computadores do hospital possam acessar as informações de seu setor e/ou de outros setores. Modificações nessas informações só podem ser feitas pelo setor da qualidade,o acesso é restritoà leitura para os demais setores do hospital.

Todos os setores do hospital possuem os seguintes documentos-padrão do SGQ: mapa de processo documento que detalha todas as entradas (inputs) do processo, os processos propriamente ditos, saídas (outputs) dos processos, material, pessoal, infraestrutura, requisitos legais e requisitos setoriais para que funcionem normalmente; matriz de risco - documento que detalha e visa prever os riscos que cada processo de determinado setor possui e se antecipar a tais riscos, planejando ações preventivas e/ou corretivas, dependendo do grau de cada um dos riscos; cadeia cliente fornecedor: documento que resguarda os gerentes de cada setor sobre acordos estabelecidos para que o mapa de processo contenha as entradas e saídas corretas, visando um processo padronizado e sem erros; procedimento operacional padrão (POP)/ procedimento sistêmico (PRS): documento que detalha a execução de determinado processo dentro do setor (POP) ou dentro de vários setores (PRS) que tem por objetivo a padronização da atividade, menor utilização de recursos materiais e humanos e minimização de erros; matriz de registro: documento no qual o gerente registra os locais de armazenamento de todo e qualquer documento que comprove realização de procedimentos, coleta de dados, estatística, dentre outros; planilha de indicadores (PIN): documento que contém indicadores gerais da rede FHEMIG e específicos de cada setor que comprove a produtividade dos servidores e que cria uma série histórica de estatísticas que podem ser utilizadas futuramente para melhorias de gestão dentro das unidades da rede.

O objetivo do sistema é que o hospital trabalhe em conjunto, aprendendo a desenvolver suas potencialidades, minimizar os erros e se tornar um ambiente próspero para os servidores.

\section{DESAFIOS DO SISTEMA DE GESTÃO DA QUALIDADE}

A trajetória da implantação do Sistema de Gestão da Qualidade no hospital- cenário, com a introdução das ferramentas de gestão e a implementação do processo, é marcada por desafios. Esses desafios devem ser tomados como oportunidade de repensar o processo assistencial, o processo de gestão e os processos decisórios internos e externos que interferem no andamento do projeto que visa a certificação da Organização Nacional de Acreditação.

A seguir, passamos a discutir os principais desafios identificados na trajetória de implantação do SGQ no Hospital.

O processo de identificação dos desafios considerou a vivência e a percepção dos componentes do Escritório da Qualidade e o não-cumprimento dos cronogramas estabelecidos para que o Hospital fosse submetido à auditoria certificadora ainda em 2010.

\section{Desafio 1: A cultura da qualidade e o papel gerencial}

O maior desafio identificado na implementação do SGQ foi conscientizar os trabalhadores de que a qualidade é inerente ao processo assistencial. Ao assumí-la os funcionários não estão fazendo algo a mais, mas agregando valor ao que já realizam. Nesse sentido, falas como "isso não faz parte das minhas atribuições" ou "o que o meu trabalho tem a ver com a qualidade" revelam desde equívocos na apropriação da qualidade como problemas na comunicação interna e transmissão do conceito aos funcionários. 


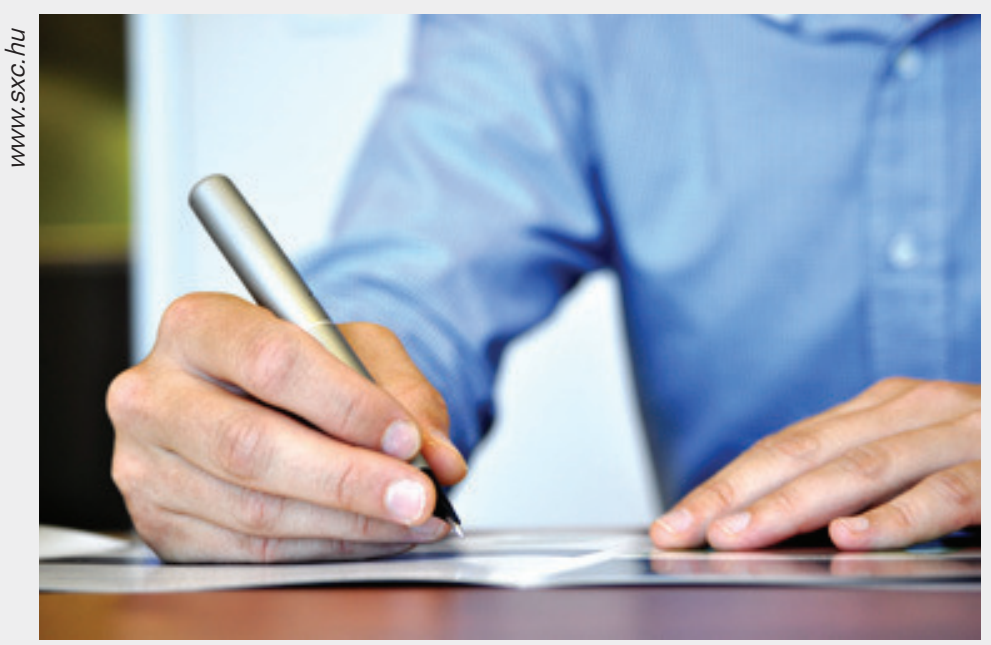

O empenho dos gerentes dos diferentes setores do Hospital diferiu durante todo o processo de implantação do Sistema de Gestão da Qualidade. Ficou claro que vários gerentes aderiram ao projeto da FHEMIG de tornar o Hospital o primeiro da rede a ser acreditado pela ONA em Belo Horizonte. Inúmeras capacitações foram feitas pela empresa de consultoria contratada pelo hospital e percebeu-se que a participação nas discussões e o envolvimento efetivo foram restritos ao mesmo grupo de gerentes.

Segundo um estudo desenvolvido por Greenfield e Braithwaite (2008), nos Estados Unidos, ainda não existem evidências concretas que justifiquem as diferentes atitudes assumidas pelos profissionais de saúde frente ao processo de acreditação, principalmente dos gerentes dos serviços. Acredita-se, porém, que os gerentes que desenvolvem os projetos em seus locais de trabalho têm capacidade de estimular o processo em outros estabelecimentos de saúde e em outros setores.

Visto dessa maneira, podemos considerar que a médio/longo prazo, os gerentes do Hospital poderão ser mais incentivados pela própria evolução de outros setores que executam suas atividades no prazo adequado e de maneira correta (efeito cascata).

No início dos trabalhos, mapeamos alguns setores onde o desconhecimento acerca do que significava a implantação do Sistema de Gestão da Qualidade ainda imperava. Esses setores foram abordados de maneira mais intensa, porém o Setor da Qualidade não dispunha de pessoal suficiente para que capacitações ocorressem com todos os funcionários. Seria, então, função do gerente do setor fazer esse contato mais direto com seus colaboradores para que dúvidas fossem sanadas e maiores explicações dadas.

Ainda assim, mesmo depois da auditoria diagnóstica pela empresa acreditadora, inúmeros funcionários (em sua grande maioria, da área assistencial) ainda procuram os estagiários e a responsável pelo Setor da Qualidade para que explicassem do que trata todo esse processo de acreditação.

Essa demanda fez com que uma atividade fosse desenvolvida dentro de alguns setores do hospital: um trabalho de conscientização. Está sendo realizado com os gerentes e seus funcionários, com o objetivo de criar um maior vínculo entre eles e a equipe de qualidade, além de colocá-los a par de tudo o que vem sendo feito e que ainda será feito para alcançar o objetivo maior do hospital que é a melhoria da qualidade da assistência.

Nesse foco, Neto e Bittar (2004) apontam que a qualidade é um processo de transformação do trabalho e do comportamento das pessoas no sentido da melhoria dos procedimentos e dos resultados da instituição. Assim, essas conquistas apenas serão possíveis por meio de capacitação coletiva, buscando tornar o trabalho mais significativo. Esse mesmo autor ressalta que as organizações hospitalares, ao focarem a qualidade do atendimento e a melhoria contínua, associadas a uma profunda mudança cultural e ao treinamento em liderança e educação continuada de todos os níveis funcionais, poderiam atender melhor seus clientes.

Manzo (2009) reforça que é imprescindível o envolvimento das pessoas na gestão da qualidade. Para que isso aconteça é fundamental, em primeiro lugar, fortalecer as pessoas pela educação continuada e capacitação permanente, pois só assim os funcionários terão segurança e senso crítico, serão criativos e avaliarão melhor os processos, dando-Ihes mais eficácia e garantindo os resultados previstos. A segunda medida necessária é o preparo das pessoas para o trabalho em equipe, o que aumenta o compromisso recíproco, incentiva a produtividade e gera um elevado nível de satisfação.

\section{Desafio2 : Descontinuidade do Trabalho e Perda do Norteamento das Ações}

A falta de acúmulo de experiências em processos anteriores dificulta uma conformação de uma cultura organizacional voltada para a qualidade. Nesse caso, a assessoria externa é uma solução que direciona as ações. Contudo, no hospital- cenário, a perda da continuidade dos serviços prestados pela empresa de consultoria retardou o seguimento dos trabalhos após a auditoria diagnóstica. Somente após quase cinco meses da auditoria diagnóstica externa, a consultoria em saúde retomou o processo junto ao Setor 
da Qualidade, verificando quais foram as não- conformidades evidenciadas e quais estão sendo as ações dos gerentes para saná-las.

Embora a descontinuidade não tenha significado supressão do trabalho do Setor de Qualidade, observamos um novo impulso com a presença dos assessores. Isso pode em parte justificar o atraso no cronograma que previa a auditoria de certificação ainda em 2010.

\section{Desafio3: Relações interpessoais e o Trabalho em Equipe}

As questões relativas a recursos humanos representam um componente crítico para o delineamento de novos paradigmas gerenciais para os serviços de saúde, em especial na área pública. Todos reconhecem que o desempenho de qualquer organização depende de seu pessoal. Esse ponto é proclamado em alto e bom som por executivos de todos os setores, sendo muito conhecidas, de todos os que trabalham no ramo da saúde, as declarações com esse teor dos dirigentes institucionais, em especial no início de suas gestões. Contudo, medidas concretas e eficazes dificilmente são operacionalizadas, seja por falta ou timidez das decisões políticas, seja porque os esquemas tradicionais não correspondem à complexidade e ao dinamismo dos problemas vigentes.

No hospital- cenário, identificamos que a co-existência de diferentes vínculos de contratação pode ser considerada um fator dificultador para a implementação do SGQ uma vez que há um "clima" entre funcionários de diferentes setores. Assim, as ferramentas que exigem construção coletiva, tais como os Procedimentos operacionais- padrão e os Procedimentos Sistêmicos, ficam prejudicadas neste processo. Ademais, a padronização de impressos também é uma etapa do processo de gestão da qualidade que exigiria um consenso e uniformidade na utilização da ferramenta.

\section{CONSIDERAÇÕES FINAIS}

O processo de acreditação está diretamente relacionado ao desenvolvimento do país e da instituição que está sendo avaliada. Portanto, podemos afirmar que o processo de acreditação na instituição relatada também está em desenvolvimento. Como parte do processo, os desafios devem ser encarados como oportunidade de crescimento. Identificamos também potencialidades que devem ser citadas:

- Disponibilidade da diretoria da FHEMIG no cumprimento de sua meta (acreditar todas as unidades em 10 anos):

- Sinergia entre planejamento da FHEMIG e instituições.

- Criação do Comitê da Qualidade na unidade em processo de acreditação formado por gerentes e colaboradores de vários setores do Hospital, com o objetivo de difundir a cultura da qualidade;

- Mudança do organograma da instituição com a criação do órgão de gestão da qualidade com atuação fundamental para o bom andamento dos trabalhos dentro da instituição.

- Manutenção da assessoria externa que atua principalmente na educação continuada dos gerentes e funcionários e apoiando o Setor da Qualidade

Visualiza-se que a trajetória, na concepção de movimento em contínua construção, não estará acabada com a certificação. A certificação não é o ponto final, mas o ponto de partida, pois apenas abre novas frentes de trabalho e permite repensar, a cada dia, a qualidade nas instituições de saúde. 


\section{6 - REFERÊNCIAS}

BALSANELLI, A. P.; JERICÓ, M. C.. Os reflexos da gestão pela qualidade total em instituições hospitalares brasileiras Acta Paulista de Enfermagem, v. 18, 2005, p. 397-402.

BRASIL. Organização Nacional de Acreditação. Manual de Organizações Prestadoras de Serviços Hospitalares. Brasília: ONA, 2004.

CORNETTA, V.K., FELICE, S.A. Desenvolvimento da qualidade: garantia da eficiência nos serviços de saúde. Laes Haes, v. 15, n. 89, 1994, p. 58-60.

D'INNOCENZO, M.; ADAMI, N. P.; CUNHA, I. C. K. O. O movimento pela qualidade nos serviços de saúde e enfermagem. Revista Brasileira de Enfermagem, v. 56, 2006, p.84-88.

FELDMAN, L. B.; GATTO, M. A. F.; CUNHA, I. C. K. O. História da evolução da qualidade hospitalar: dos padrões a acreditação. Acta Paulista de Enfermagem, 2005; v. 18, 2005, p. 213-219.

FERREIRA, F. G. Desenvolvimento e aplicação de um de programa da qualidade para o serviço público. 1999. 230f. Dissertação (Mestrado em Engenharia) - Programa de Pós-Graduação em Engenharia de Produção e Sistemas, Universidade Federal de Santa Catarina, Santa Catarina. Disponível em: <http://www.eps.ufsc.br/disserta99/ferreira/cap.html> Acesso em: 04 de Setembro de 2010 às $15: 48$

FUNDAÇÃO HOSPITALAR DO ESTADO DE MINAS GERAIS. Cartilha do Processo de Acreditação. FHEMIG, 2010

FUNDAÇÃO HOSPITALAR DO ESTADO DE MINAS GERAIS. Planejamento Estratégico FHEMIG 20082018. FHEMIG, 2008

HOUAISS. Grande dicionário da lingual portuguesa, página 2345, 2001.LAS CASAS, A. L. Marketing de serviços. 2. ed. São Paulo: Atlas, 2000.

MANZO, B. F.. O Processo de Acreditação Hospitalar na perspectiva de profissionais de saúde. 2009. 98 f. Dissertação (Mestrado em Saúde e Enfermagem) - Escola de Enfermagem, Universidade Federal de Minas Gerais, Belo Horizonte, 2009.

NETO, A. Q.; BITTAR, O. J. N. Hospitais: administração da qualidade e Acreditação de organizações complexas. 1. ed. Porto Alegre: Dacasa, 2004. 315 p.

NOVAES, H. M.; PAGANINI, J. M. Garantia da qualidade em hospitais da América Latina e do Caribe: Acreditação de hospitais para América Latina e do Caribe. Organização Pan Americana de hospitais/ Organização Mundial de Saúde/Federação Latina Americana de Hospitais. Brasília (DF): editora da Federação Brasileira de Hospitais, 1994.

ORGANIZAÇÂO NACIONAL DE ACREDITAÇÃO (ONA). A saúde no Brasil: agora tem um processo permanente de avaliação e certificação da qualidade. Brasília (DF): 2000. Folder.

ORGANIZAÇÂO NACIONAL DE ACREDITAÇÃO (ONA). Organizações certificadas. 2012. Disponível em: https://www.ona.org.br/OrganizacoesCertificadas/0 [Acesso em 10 jan 2012].

ROONEY, A. L.; OSTENBER, P. R. Licenciamento, Acreditação e certificação: abordagens à qualidade de serviços de saúde. Projeto de garantia da qualidade, Centro dos serviços Humanos - CHS. USA: USAID, 1999. 64 p. 